\title{
Commentary: A therapeutic Jedi mind trick: A neuroprotective target to counteract deep hypothermic circulatory arrest
}

\author{
Nicholas D. Andersen, MD, Mohammed K. Alsarraj, BS, and Joseph W. Turek, MD, PhD
}

\author{
From the Duke Congenital Heart Research \& Training Laboratory, Duke University, Durham, NC. \\ Disclosures: Authors have nothing to disclose with regard to commercial support. \\ Received for publication June 28, 2019; accepted for publication June 28, 2019; available ahead of print Aug 13, \\ 2019. \\ Address for reprints: Joseph W. Turek, MD, PhD, Division of Cardiovascular and Thoracic Surgery, Duke \\ University Medical Center, 2301 Erwin Rd, DUMC 3474, Durham, NC 27710 (E-mail: joseph.turek@duke. \\ edu). \\ J Thorac Cardiovasc Surg 2020;159:2445-6 \\ 0022-5223/ $\$ 36.00$ \\ Copyright (C) 2019 by The American Association for Thoracic Surgery \\ https://doi.org/10.1016/j.jtcvs.2019.06.082
}

Neuroinflammation promoting neuronal damage after deep hypothermic circulatory arrest (DHCA) has been well characterized. ${ }^{1,2}$ Unfortunately, finding a remedy has remained a challenge, with underlying molecular mechanisms not clearly delineated. In the current issue of the Journal, Liu and colleagues ${ }^{3}$ have described the possible role of coldinducible RNA-binding protein (CIRP) in promoting neuroinflammation after DHCA. Specifically, under conditions of deep hypothermia, oxidative stress, and hypoxia lead to overactivation of microglial cells in the brain, promoting increased proinflammatory gene transcription and subsequent neuronal injury (Figure 1).

Liu and colleagues ${ }^{3}$ were able to identify bromodomain and extra terminal domain proteins, such as the transcriptional activator Brd2, as key mediators of the inflammatory response induced by microglial cells. The experimental strategy used an established rat model of hypothermia and hypoxia with normal and CIRP knockout animals. ${ }^{4}$ The results showed CIRP levels to be significantly increased in the normal, DHCA-treated group compared with the sham group. The neuronal injury inflicted by CIRP in the normal, DHCA group demonstrated characteristics of ischemic cell death, as well as elevated cerebral injury markers in the plasma, such as tumor necrosis factor- $\alpha$, interleukin-5, and interleukin-13, that were not present in the DHCA, CIRP knockout rats. Through RNA-sequencing analysis, it was shown that the expression of the protein $\mathrm{Brd} 2$ was greatly reduced in the hippocampus of DHCA, CIRP knockout rats.

An inhibitor of bromodomain and extra terminal domain proteins (JQ1) was administered that blocks the acetylated lysine binding pockets, which limits the downstream effects of the proinflammatory cytokines released by microglial cells. Under experimental conditions of hypothermia and oxidative stress, both $\operatorname{Brd} 2$ proteins and the nuclear factor-kB proinflammatory pathway were significantly downregulated upon CIRP removal. Thus, the inhibition

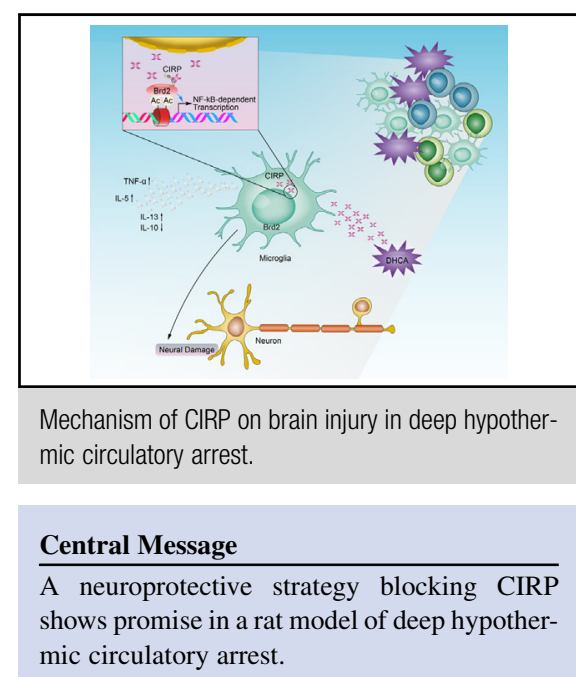

See Article page 2431 .

of the nuclear factor-kB transcription pathway in microglia through the inhibition of CIRP activity can lead to the downregulation of neuronal damage, and therefore may have therapeutic implications in treating DHCA-induced cerebral injury.

This article offers compelling support for CIRP, largely through the Brd2 protein, as a pivotal initiator of microglial activation, inflammatory gene transcription, and the devastating effects of neuroinflammation after exposure to DHCA. Furthermore, this knowledge introduces promising therapeutic targets (inhibitors of CIRP and Brd2) to "trick" microglia into not activating, thereby protecting the mind from inflammation-related neuronal damage during deep hypothermia. In essence, this strategy tells microglia, "these aren't the neurons you're looking for."

\section{References}

1. Zhang Z, Ma Q, Shah B, Mackensen GB, Lo DC, Mathew JP, et al. Neuroprotective effects of annexin A1 tripeptide after deep hypothermic circulatory arrest in rats. Front Immunol. 2017;8:1050.

2. Kellerman K, Dertinger N, Blobner M, Kees F, Kochs EF, Jungwirth B. Perioperative moxifloxacin treatment in rats subjected to deep hypothermic circulatory arrest: reduction in cerebral inflammation but without improvement in cognitive performance. J Thorac Cardiovasc Surg. 2011;141:796-802.

3. Liu M, Li Y, Gao S, Yan S, Zhang Q, Liu G, et al. A novel target to reduce microglial inflammation and neuronal damage after deep hypothermic circulatory arrest. J Thorac Cardiovasc Surg. 2020;159:2431-44.e7.

4. Liu M, Zeng Q, Li Y, Liu G, Ji B. Neurologic recovery after deep hypothermic circulatory arrest in rats: description of a long-term survival model without blood priming. Artif Organs. 2019;43:551-60. 


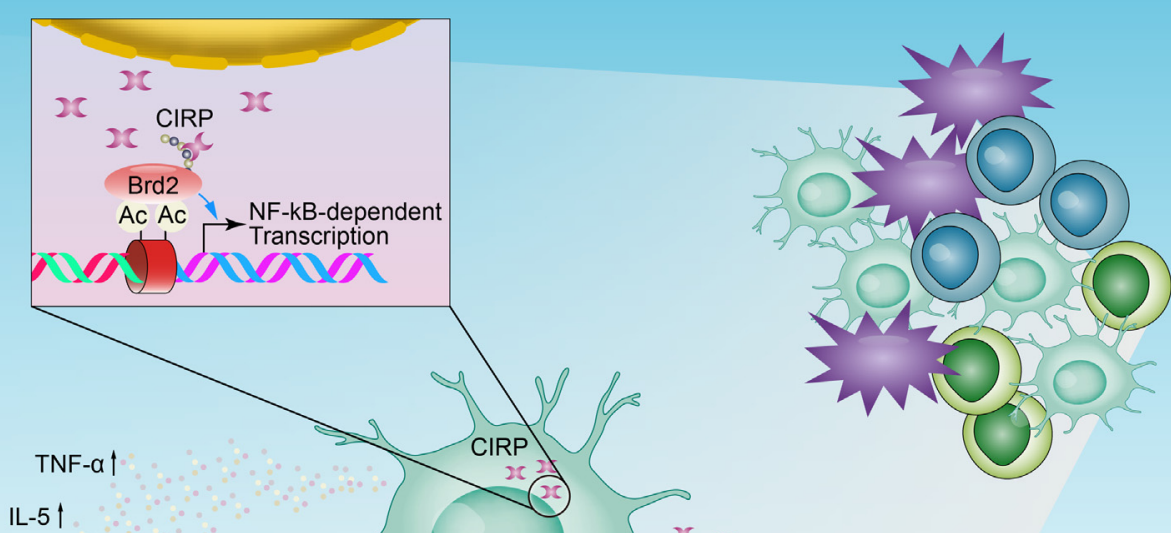

$\mathrm{IL}-13 \uparrow$

IL-10 ।

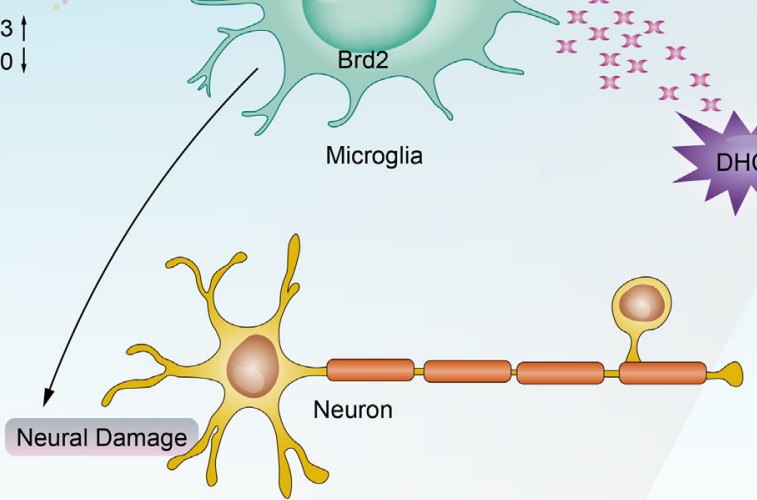

FIGURE 1. Mechanism of cold-inducible RNA-binding protein on brain injury in deep hypothermic circulatory arrest. 